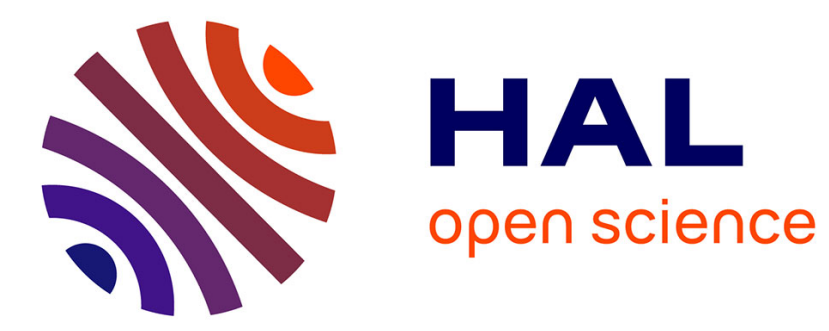

\title{
Fast Radio Broadcasting with Advice
}

\author{
David Ilcinkas, Dariusz R. Kowalski, Andrzej Pelc
}

\section{To cite this version:}

David Ilcinkas, Dariusz R. Kowalski, Andrzej Pelc. Fast Radio Broadcasting with Advice. SIROCCO 2008, Jun 2008, Villars-sur-Ollon, Switzerland. pp.291-305, 10.1007/978-3-540-69355-0_24 . hal00341449

\section{HAL Id: hal-00341449 \\ https://hal.science/hal-00341449}

Submitted on 25 Nov 2008

HAL is a multi-disciplinary open access archive for the deposit and dissemination of scientific research documents, whether they are published or not. The documents may come from teaching and research institutions in France or abroad, or from public or private research centers.
L'archive ouverte pluridisciplinaire $\mathbf{H A L}$, est destinée au dépôt et à la diffusion de documents scientifiques de niveau recherche, publiés ou non, émanant des établissements d'enseignement et de recherche français ou étrangers, des laboratoires publics ou privés. 


\title{
Fast Radio Broadcasting with Advice
}

\author{
David Ilcinkas ${ }^{1, \star}$, Dariusz R. Kowalski ${ }^{2}$, and Andrzej Pelc ${ }^{3, \star \star}$ \\ 1 CNRS, LaBRI, Université Bordeaux I, France \\ david.ilcinkas@labri.fr \\ 2 Department of Computer Science, The University of Liverpool, United Kingdom \\ darek@csc.liv.ac.uk \\ 3 Département d'informatique, Université du Québec en Outaouais, Canada. \\ pelc@uqo.ca
}

\begin{abstract}
We study deterministic broadcasting in radio networks in the recently introduced framework of network algorithms with advice. We concentrate on the problem of trade-offs between the number of bits of information (size of advice) available to nodes and the time in which broadcasting can be accomplished. In particular, we ask what is the minimum number of bits of information that must be available to nodes of the network, in order to broadcast very fast. For networks in which constant time broadcast is possible under complete knowledge of the network we give a tight answer to the above question: $O(n)$ bits of advice are sufficient but $o(n)$ bits are not, in order to achieve constant broadcasting time in all these networks. This is in sharp contrast with geometric radio networks of constant broadcasting time: we show that in these networks a constant number of bits suffices to broadcast in constant time. For arbitrary radio networks we present a broadcasting algorithm whose time is inverse-proportional to the size of advice.

Key words: radio network, distributed algorithm, deterministic broadcasting, advice
\end{abstract}

\section{Introduction}

\section{The Framework and the Problem.}

We study deterministic broadcasting in radio networks in the recently introduced [17] framework of network algorithms with advice. This paradigm permits to investigate the minimum amount of information (size of advice) that nodes of the network have to be given in order to accomplish some distributed task with a given efficiency. In our present context the task is broadcasting in radio networks and the measure of efficiency is time.

A radio network is a collection of sites (stations) equipped with wireless transmission and receiving capabilities, with a distinguished node $s$ called the

* This work was done during the stay of David Ilcinkas at the Research Chair in Distributed Computing of the Université du Québec en Outaouais and at the University of Ottawa, as a postdoctoral fellow.

** Research partially supported by NSERC discovery grant and by the Research Chair in Distributed Computing at the Université du Québec en Outaouais. 
source. The topology of a radio network is modeled as a directed graph $G=$ $(V, E)$, where nodes in $V$ represent sites of the network and oriented edges in $E$ correspond to wireless connections. It is assumed that there is a directed path from the source to every other node. The existence of an edge $(u, v)$ means that $v$ is within the reach of $u$. We say that $u$ is an in-neighbor of $v$ and $v$ is an out-neighbor of $u$. Nodes that are not neighbors must communicate via intermediate (relaying) nodes. Similarly as in most papers in the literature on radio networks, we assume that communication is synchronous, i.e., all nodes have internal clocks that tick at the same rate, measuring consecutive time steps, referred to as rounds. All clocks show the same round number at any given time.

At any round every node can be either in the transmitting or in the receiving mode, i.e., a node cannot transmit and receive messages during the same round. When a node $v$ transmits in round $i$, its message is delivered during this round to all out-neighbors of $v$. However, if $w$ is an out-neighbor of $v$, this message is heard by $w$, i.e., $w$ receives the message correctly, if and only if the node $v$ is the only in-neighbor of $w$ that transmits during the round $i$. Otherwise a collision occurs at $w$ and the message is not heard. An important property of radio networks is the collision detection capability, i.e., the ability of a node to differentiate collision from silence in a given round. All our results hold both with this assumption and without it. Indeed, our positive results (algorithms) are valid even without collision detection, and our impossibility results are valid even assuming this capability.

Among the large class of (arbitrary) radio networks, an important subclass consists of geometric radio networks (GRN). In the case of an approximately flat region without large obstacles, nodes that can be reached from $u$ are those within a circle of radius $r$ centered at $u$, and the positive real $r$, called the range of $u$, depends on the power of the transmitter located at $u$. Reachability graphs corresponding to such radio networks are called geometric radio networks. More precisely, they are defined as follows. We assume that there is a constant number $\rho$ of possible powers of transmitters, thus we fix a set $R=\left\{r_{1}, \ldots, r_{\rho}\right\}$ of positive reals, $r_{1}<\ldots<r_{\rho}$, called ranges. Let $C$ be a set of points in the plane with a distinguished source. Points of $C$ are nodes of the graph (representing radio stations). Each point $u \in C$ is assigned a range $r(u) \in R$ and a directed edge $(u, v)$ exists in the graph, if and only if the Euclidean distance between $u$ and $v$ does not exceed $r(u)$.

The number of nodes of a radio network is denoted by $n$, and the eccentricity of the source (the maximum length of all shortest paths in the graph from the source to all other nodes) is denoted by $D$. Throughout the paper, log denotes the logarithm with base 2 and $\ln$ denotes the natural logarithm. Nodes of a radio network have distinct labels from the set $\{1, \ldots, N\}$, where $N \in O(n)$. Moreover, nodes of a geometric radio network have also their $(x, y)$ coordinates. A priori, each node of a (general) radio network knows only its own label, and each node of a GRN knows only its own label and its $(x, y)$ coordinates, as well as the set $R$ of available ranges (which has constant size). All other information about the network must be given to nodes as advice, to be defined below. 
One of the most studied communication primitives in networks is broadcasting, also known as one-to-all communication. The source has a message that should be distributed to all other nodes in the network. The time of a deterministic broadcasting algorithm is the number of rounds in which all the nodes get the source message. With every radio network $G$ we associate its optimal broadcasting time $\operatorname{Opt}(G)$. This is the minimum time in which broadcasting in this network can be accomplished, if nodes have full information about the network. Establishing optimal broadcasting time for a given radio network is an NP-hard problem [5].

It remains to formalize the framework of advice (cf. [17]) in our present context. All additional knowledge available to the nodes of the network (in particular knowledge concerning the rest of the network), is modeled by an oracle providing advice. An oracle is a function $\mathcal{O}$ whose arguments are labeled networks (in the case of geometric radio networks these arguments are actual sets of points in the plane, together with the assigned ranges and labels), and the value $\mathcal{O}(G)$, for a network $G=(V, E)$, called the advice provided by the oracle to this network, is in turn a function $f: V \rightarrow\{0,1\}^{*}$ assigning a binary string to every node $v$ of the network. Intuitively, the oracle looks at the entire labeled network and assigns to every node some information, encoded as a string of bits. The size of the advice given by the oracle to a given network $G$ is the sum of the lengths of all the strings it assigns to nodes. Hence this size is a measure of the amount of information about the network, available to its nodes. Solving the broadcasting problem in radio networks using advice provided by oracle $\mathcal{O}$ consists in designing an algorithm that is unaware of the network $G$ at hand but accomplishes broadcasting in it, as long as every node $v$ of the network $G$ is provided with the string of bits (advice) $f(v)$, where $f=\mathcal{O}(G)$.

The main interest of this framework is the significance of lower bounds on the size of advice. If we have a broadcasting algorithm using some advice of size $O(g(n))$ and achieving time $O(T(n))$, in $n$-node networks, and at the same time we prove that $\Omega(g(n))$ is the lower bound on the size of advice needed to achieve time $O(T(n))$, this implies optimality in a very strong sense: smaller amount of information of any type cannot help to achieve broadcasting time $O(T(n))$ using any algorithm. In other words, changing the type of information provided to nodes cannot help to achieve the same efficiency of broadcasting at lower information cost.

This paper is the first to consider communication in radio networks in the framework of algorithms with advice. Our research is motivated by the following problems:

- What is the minimum size of advice permitting to achieve broadcasting time $O(O p t(G))$ for a radio network $G$ ?

- What are the trade-offs between the size of advice and the time of broadcasting in radio networks?

\section{Our Results.}

Our main focus is on radio networks with constant optimal broadcasting time, i.e., on networks in which deterministic broadcast in constant time is possible 
under complete knowledge of the network. For this class of networks we establish the minimum size of advice sufficient to achieve constant broadcasting time. We show that $O(n)$ bits of advice are sufficient and $o(n)$ bits are not sufficient, in order to achieve constant broadcasting time in all these networks. The main contribution of this part of the paper is the above tight lower bound on the size of advice. This is in sharp contrast with geometric radio networks of constant broadcasting time: we show that in these networks a constant number of bits of advice suffices to broadcast in constant time.

For arbitrary radio networks we show a trade-off between the size of advice and the time of deterministic broadcasting, by presenting a broadcasting algorithm whose time is inverse-proportional to the size of advice. More precisely, for any $q \in O(n)$ we show an oracle which gives advice of size $q$ to the nodes of a network, and an algorithm using this advice, which performs broadcasting in time $O\left(\frac{n D}{q} \log ^{3} n\right)$ in any $n$-node network with source eccentricity $D$. As a corollary we get that for "short" networks, i.e., with $D$ polylogarithmic in $n$, an advice of sublinear size suffices to achieve polylogarithmic broadcasting time.

\section{Related Work.}

The paradigm of distributed computing with advice has been recently introduced in [17] and used there to study the task of broadcasting with a linear number of messages, in the message passing model. Subsequently, this approach has been used in [18] to study efficient exploration of networks by mobile agents, in [19] to study distributed graph coloring, in [20] to study the distributed minimum spanning tree construction, and in [30] to study graph searching.

Broadcasting in radio networks is a topic extensively studied in the last twenty years. Most of the papers represented radio networks as arbitrary (undirected or directed) graphs. Models used in the literature about algorithmic aspects of radio communication, starting from the paper [5], differ mostly in the amount of information about the network that is assumed available to nodes. However, assumptions about this knowledge concern particular items of information, such as the knowledge of the size of the network, its diameter, maximum degree, or some neighborhood around the nodes, rather than limiting the total number of bits available to nodes, regardless of their meaning, as is the case with the advice approach.

Deterministic centralized broadcasting assuming complete knowledge of the network was considered, e.g., in [6], where a polynomial-time algorithm constructing a $O\left(D \log ^{2} n\right)$-time broadcasting scheme was given for all $n$-node networks of radius $D$. Subsequent improvements by many authors $[15,21,22]$ were followed by the polynomial-time algorithm from [27] constructing a $O(D+$ $\log ^{2} n$ )-time broadcasting scheme, which is optimal. On the other hand, in [1] the authors proved the existence of a family of $n$-node networks of radius 2 , for which any broadcast requires time $\Omega\left(\log ^{2} n\right)$.

One of the first papers to study deterministic distributed broadcasting in radio networks whose nodes have only limited knowledge of the topology, was [2]. The authors assumed that nodes know only their own label and labels of their neighbors. Many authors $[4,7,8,10]$ studied deterministic distributed broadcast- 
ing in radio networks under the assumption that nodes know only their own label (but not labels of their neighbors). Increasingly faster broadcasting algorithms working on arbitrary radio networks were constructed, the currently fastest being the $O\left(n \log ^{2} D\right)$-time algorithm from [11] and the $O(n \log n \log \log n)$ algorithm from [12]. On the other hand, in [10] a lower bound $\Omega(n \log D)$ on broadcasting time was proved for $n$-node networks of radius $D$.

Randomized broadcasting algorithms in radio networks were studied, e.g., in $[2,28,26]$. For these algorithms, no topological knowledge of the network and no distinct identities of nodes were supposed.

Broadcasting in geometric radio networks and some of their variations was considered, e.g., in $[13,14,29]$. In [29] the authors proved that scheduling optimal broadcasting is NP-hard even when restricted to such graphs, and gave an $O(n \log n)$ algorithm to schedule an optimal broadcast when nodes are situated on a line. In [14] broadcasting with restricted knowledge was considered but the authors studied only the special case of nodes situated on the line. In [13], the authors investigated the impact of the size of the part of the geometric radio network known to nodes, on the efficiency of broadcasting. In particular they showed that with the full knowledge of the network broadcasting can be accomplished in (optimal) time $O(D)$, and if all nodes know only their own label, range and coordinates, broadcasting in time $O(n)$ is possible. For symmetric geometric radio networks, time $O(D+\log n)$ was proved optimal under this restricted knowledge, if collision detection is available. If it is not, the same broadcasting time was achieved if nodes know positions, labels and ranges of all nodes within a constant (arbitrarily small) positive radius. In a recent paper [16] the authors considered broadcasting in radio networks represented by unit disk graphs. They compared broadcasting time in two models: the model allowing spontaneous transmissions of nodes that have not yet gotten the source message, and the model in which only nodes that already obtained the source message can transmit.

\section{Broadcasting in Constant Time}

In this section we focus on radio networks with constant optimal broadcasting time, i.e., on the class of networks in which broadcasting in constant time is possible if nodes have complete knowledge of the network. Such networks must of course have bounded source eccentricity $D$. However, this is not a sufficient condition. Indeed, there are $n$-node networks with $D=2$, whose minimum broadcasting time is $\Omega\left(\log ^{2} n\right)$, even if the network is completely known to all nodes (cf. [1]).

Networks with constant optimal broadcasting time may require a very long broadcasting time if their topology is unknown and in the absence of any advice. In [25] a family of such $n$-node networks was proved to require time $\Omega(n)$. In fact, even for the more restricted class of geometric radio networks, strong lower bounds of this type can be proven. Using techniques from [16] a class of geometric radio networks with constant optimal broadcasting time can be shown to require 
time $\Omega(\sqrt{n})$, if nodes know only their own label and coordinates. Therefore it is natural to ask how sensitive to advice is broadcasting time in networks (geometric or not) with constant optimal broadcasting time. More precisely, how much advice is needed to achieve constant broadcasting time in such networks.

First observe that for networks of the considered class, $O(n)$ bits of advice are sufficient in order to achieve constant broadcasting time.

Proposition 1. For any positive constant $c$ let $\mathcal{C}$ be the class of $n$-node radio networks whose optimal broadcasting time is at most $c$. There exists an oracle which gives advice of size $O(n)$ to the nodes of networks of class $\mathcal{C}$ and an algorithm using this advice, which performs broadcast in time at most $c$, for any network in class $\mathcal{C}$.

Proof. Fix a network $C \in \mathcal{C}$ and consider an algorithm having complete knowledge of the network and broadcasting in time at most $c$. For any fixed node $v$ of $C$, let $t_{1}, \ldots, t_{k}$ be numbers of rounds in which $v$ has to transmit, according to this algorithm. The oracle gives this information, encoded as a string of bits of bounded length, to node $v$. Hence the total size of advice is $O(n)$. Now the broadcasting algorithm simply makes node $v$ transmit in rounds $t_{1}, \ldots, t_{k}$.

\subsection{Lower Bounds}

The main result of this section shows that the above upper bound on the size of advice needed to achieve constant broadcasting time is tight, i.e., that $o(n)$ bits of advice are not sufficient to broadcast in constant time.

Theorem 1. For every integer function $k^{*} \in o(n)$ there exist an integer function $c^{*}$ such that $c^{*}(n) \rightarrow \infty$ and a family of n-node networks with constant optimal broadcasting time, such that every algorithm using at most $k^{*}(n)$ bits of advice requires time $c^{*}(n)$ on some of them, for sufficiently large $n$.

We will use the following lemmas whose proofs are omitted.

Lemma 1. If $k^{*} \in o(k)$ then for any integer $0 \leq \ell \leq k^{*}$ and for sufficiently large $k$

$$
\left(\begin{array}{c}
k \\
k^{*}-\ell
\end{array}\right) \leq e^{-2 \ell} \cdot\left(\frac{k e}{k^{*}}\right)^{k^{*}} .
$$

Lemma 2. Let $x, x_{1}, \ldots, x_{a}$ be non-negative integers satisfying $x \geq x_{1} \geq \ldots \geq$ $x_{a} \geq 0$ and $x_{1}+\ldots+x_{a}=2 x$, where $2 \leq a \leq x$. The number of permutations of the set $X=\{1, \ldots, 2 x\}$ satisfying the following condition:

for any $1 \leq i \leq a$ and $1 \leq j \leq x$, no two elements from the interval $X_{i}=\left[1+\sum_{i^{\prime}=1}^{i-1} x_{i^{\prime}}, \sum_{i^{\prime}=1}^{i} x_{i^{\prime}}\right]$ are placed in positions $2 j-1,2 j$ (called group $j$ )

is at most

$$
\beta(2 x, a)=\sqrt{2} \cdot(2 x) ! \cdot e^{2 a^{2} \ln (2 e x / a)-x / a} .
$$




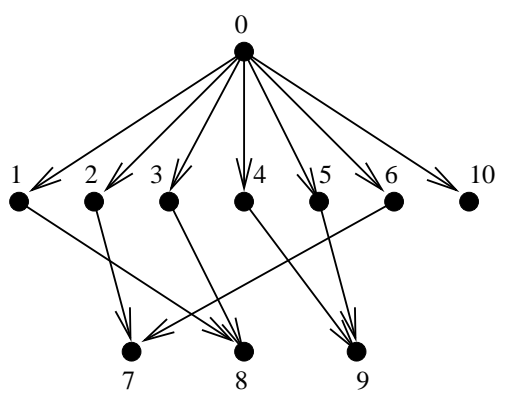

(a)

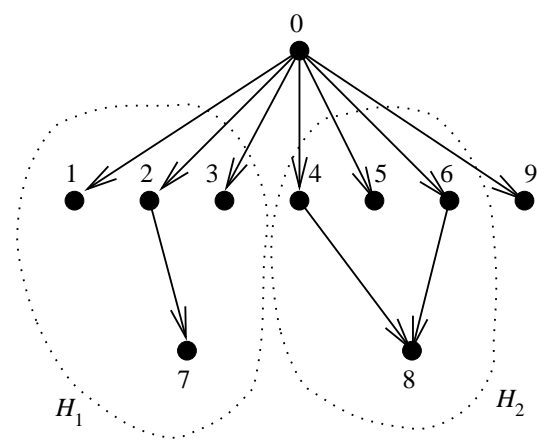

(b)

Fig. 1. (a) Example of the network in the class used in the proof of Theorem 1, for $n=11$ and $k=3$; (b) Example of the network in the class used in the proof of Theorem 2, for $n=10, n^{\prime}=3, k=2, S_{1}=\{2\} \subseteq\{1,2,3\}$ and $S_{2}=\{4,6\} \subseteq\{4,5,6\}$

Proof of Theorem 1. Fix $n$. Consider the following family $\mathcal{C}$ of $n$-node directed networks, see Fig. 1(a). Let $k=\lfloor(n-1) / 3\rfloor$. (We omit parameter $n$ from the arguments of functions $k, k^{*}, c^{*}$ since it is known from the context.) Each network in $\mathcal{C}$ is composed of three layers. Layer $L_{0}$ consists of the source with label 0 . Layer $L_{1}$ consists of $2 k$ nodes with labels from $\{1, \ldots, 2 k\}$, partitioned into $k$ disjoint groups of size 2 , and of $n-(3 k+1)$ nodes with labels from $\{3 k+1, \ldots, n\}$. Layer $L_{2}$ consists of $k$ nodes with labels from $\{2 k+1, \ldots, 3 k\}$. All nodes of layer $L_{1}$ are out-neighbors of the source, and each node $2 k+i$ from layer $L_{2}$ is the outneighbor of both nodes from the $i$ th group. There are no other edges in networks from $\mathcal{C}$. There are $\alpha=(2 k) ! / 2^{k}$ different networks in this family. The optimal broadcasting time of any network from $\mathcal{C}$ is clearly 2 . Let $c^{*}=\log \frac{k-k^{*}}{2 k^{*} \log \left(k e / k^{*}\right)}$. Clearly, $c^{*} \in \omega(1)$ for $k^{*} \in o(k)$. In view of $k=\lfloor(n-1) / 3\rfloor$, it is enough to prove that every algorithm using at most $k^{*} \in o(k)$ bits of advice requires time larger than $c^{*}$ on some network in $\mathcal{C}$. We fix $n$ such that $k$ is sufficiently large for the purpose of Lemma 1 and Fact 1, and assume that $k^{*} \in o(k)$ and $k^{*} \in \omega\left(k^{4 / 5}\right)$ (if we show that the time is $\omega(1)$ for functions $k^{*} \in \omega\left(k^{4 / 5}\right)$, the same remains true also for all smaller functions $k^{*}$ ).

The proof is by contradiction. Fix an oracle giving advice of size at most $k^{*}$ to networks from the family $\mathcal{C}$, and an algorithm using this oracle and completing the broadcast in all these networks in time at most $c^{*}$. Let $\mathcal{C}(\ell)$ be the subfamily of $\mathcal{C}$ for which the oracle gives $\ell$ bits of advice to the source, for $0 \leq \ell \leq k^{*}$, and gives the remaining bits to some other nodes of the network. For a sequence $\hat{y}$ of $\ell$ bits, let $\mathcal{C}(\ell)_{\hat{y}}$ be the subfamily of $\mathcal{C}(\ell)$ containing those networks for which the oracle gives the advice $\hat{y}$ to the source.

Fix $0 \leq \ell \leq k^{*}$. There are $2^{\ell}$ possible advice sequences in the source, and at least $k-k^{*}+\ell$ groups none of whose nodes has any advice. We call these groups blind groups. Fix a sequence $\hat{y}$ of $\ell$ bits of advice in the source and consider the transmission sequence of length at most $c^{*}$ for each node in $\{1, \ldots, 2 k\}$ assuming 
that it has no bit of advice and that the source has the advice $\hat{y}$. Formally, the transmission sequences can be longer, but it is sufficient to consider only prefixes of length at most $c^{*}$ for the purpose of proving the lower bound. Under this assumption, each node in $L_{1}$ without any advice has a fixed 0-1 transmission sequence of length $c^{*}$, since no feedback is possible, due to the absence of directed cycles in the graph. (In a transmission sequence, 0 in position $i$ means that the node does not transmit in round $i$, and 1 means that it transmits.) This yields a partition of nodes $\{1, \ldots, 2 k\}$ into at most $a=2^{c^{*}}=\frac{k-k^{*}}{2 k^{*} \log \left(k e / k^{*}\right)}$ colors, where all nodes of the same color follow the same transmission pattern during the first $c^{*}$ rounds. Note that $a^{4} \in o\left(k^{*}\right)$, by the assumption $k^{*} \in \omega\left(k^{4 / 5}\right)$. In every network in $\mathcal{C}(\ell)_{\hat{y}}$, nodes of every blind group must have different colors, because otherwise both nodes would follow the same pattern of transmissions and their out-neighbor would not receive the source message by round $c^{*}$. Therefore, the number of networks in class $\mathcal{C}(\ell)_{\hat{y}}$ is at most

$$
\left(\begin{array}{c}
k \\
k^{*}-\ell
\end{array}\right)\left(\begin{array}{c}
2 k \\
2\left(k^{*}-\ell\right)
\end{array}\right) \frac{\left(2 k^{*}-2 \ell\right) !}{2^{k^{*}-\ell}} \cdot 2^{-\left(k-k^{*}+\ell\right)} \cdot \beta\left(2 k-2 k^{*}+2 \ell, a\right),
$$

where $\beta(2 x, a)$ is the upper bound from Lemma 2 . In the above formula the first factor corresponds to the number of choices of non-blind groups (and possibly some blind ones, since the number of non-blind groups is at most $k^{*}-\ell$ ) among all groups. The second factor corresponds to the number of choices of the $2\left(k^{*}-\ell\right)$ elements to be allocated to the above groups. The third factor corresponds to the number of ways of allocating these elements to these groups. The last two factors form an upper bound on the number of different configurations of the remaining nodes such that the remaining (blind) groups are not monochromatic (i.e., the number of permutations without remaining monochromatic blind groups, divided by the number of possible flips of elements inside those groups - there are $2^{k-k^{*}+\ell}$ such flips).

Using Lemma 2 and the properties $k^{*} \in \omega\left(k^{4 / 5}\right)$ and $a^{4} \in o\left(k^{*}\right)$, we get the following fact whose proof is omitted.

Fact 1. For sufficiently large $k$,

$$
\left|C(\ell)_{\hat{y}}\right| \leq\left(\begin{array}{c}
k \\
k^{*}-\ell
\end{array}\right) \cdot \alpha \cdot e^{-k^{*} \log \left(k e / k^{*}\right)-1} .
$$

Finally, using Fact 1 and Lemma 1, we can bound

$$
\begin{aligned}
|C| & =\sum_{\ell=0}^{k^{*}} \sum_{\hat{y}}\left|C(\ell)_{\hat{y}}\right| \leq \sum_{\ell=0}^{k^{*}} \sum_{\hat{y}}\left(\alpha \cdot\left(\begin{array}{c}
k \\
k^{*}-\ell
\end{array}\right) \cdot e^{-k^{*} \log \left(k e / k^{*}\right)-1}\right) \\
& \leq \sum_{\ell=0}^{k^{*}}\left(2^{\ell} \cdot \alpha \cdot e^{-2 \ell} \cdot e^{k^{*} \ln \left(k e / k^{*}\right)} \cdot e^{-k^{*} \log \left(k e / k^{*}\right)-1}\right) \\
& \leq(\alpha / e) \cdot \sum_{\ell=0}^{k^{*}} e^{-\ell}<\alpha
\end{aligned}
$$


for sufficiently large $k$. This is a contradiction which completes the proof of Theorem 1.

Our next result shows that if the advice is of sublogarithmic size then the time required for broadcasting is not only unbounded but sometimes quite large.

Theorem 2. Fix any constant $\delta<1$. There exists a constant $c>0$ such that, for sufficiently large $n$, there exists a family of n-node networks with constant optimal broadcasting time, for which every algorithm using at most $c \log n$ bits of advice requires time at least $n^{\delta}$ on some of them.

Proof. Fix any $0<\delta<1$. For a positive integer $n$, we set $n^{\prime}=\left\lceil n^{\delta}\right\rceil$ and $k=\left\lfloor\frac{n-1}{n^{\prime}+1}\right\rfloor$. For $n$ large enough, there exists $0<\epsilon<1$ such that $k \geq n^{\epsilon}$. For any $k$-tuple $\mathcal{S}=\left(S_{1}, S_{2}, \ldots, S_{k}\right)$, where each $S_{i}, 1 \leq i \leq k$, is an arbitrary nonempty subset of $\left\{1, \ldots, n^{\prime}\right\}$, we define the directed graph $G_{\mathcal{S}}$ as follows. The source is node 0 . It has directed edges to $k \cdot n^{\prime}$ nodes labelled from 1 to $k \cdot n^{\prime}$. For any $1 \leq i \leq k$, if $j \in S_{i}$ then node $(i-1) n^{\prime}+j$ has a directed edge to node $k \cdot n^{\prime}+i$. Finally, in order to have exactly $n$ nodes, the source has directed edges to the nodes from $k\left(n^{\prime}+1\right)+1$ to $n-1$, if any. Hence the graph has $k$ disjoint $\left(n^{\prime}+1\right)$-node subgraphs $H_{1}, \ldots, H_{k}$, attached to the source. More precisely, the subgraph $H_{i}$ is induced by the nodes $(i-1) n^{\prime}+1, \ldots, i \cdot n^{\prime}, k \cdot n^{\prime}+i$. The directed edges inside a subgraph $H_{i}$ are determined by the set $S_{i}$. The set of graphs $G_{\mathcal{S}}$, for all possible $\mathcal{S}$, is denoted $\mathcal{G}$. See Fig. 1(b).

We prove that there is no algorithm using advice of size $q \leq \frac{1}{2} \log k$ that achieves broadcast in the family $\mathcal{G}$ in time smaller than $n^{\prime}$. Fix an algorithm using advice of size $q \leq \frac{1}{2} \log k$. Let $s_{1}, \ldots, s_{Q}$, for $Q=2^{q+1}-1$ be an enumeration of all binary sequences of length at most $q$ (including the empty sequence). First note that $Q \cdot(q+1) \leq k$, for sufficiently large $n$. Consider the following property:

For any $1 \leq i \leq Q \cdot(q+1)$, there exists a non-empty subset $S_{i}$ of $\left\{1, \ldots, n^{\prime}\right\}$ such that for any $k$-tuple $\mathcal{S}$ containing $S_{i}$ as the $i$-th element we have that, in the graph $G_{\mathcal{S}}$, either

(1) the source has advice different from $s_{j}$, where $j=\left\lfloor\frac{i-1}{q+1}\right\rfloor$, or

(2) at least one node of the subgraph $H_{i}$ receives at least one bit of advice.

This implies that for a $k$-tuple $\mathcal{S}$ such that the $Q \cdot(q+1)$ first elements are the above mentioned sets $S_{i}$, there exist at least $q+1$ different subgraphs $H_{i}$ receiving at least one bit. Indeed, if the advice given to the source is $s_{j}$, each of the graphs $H_{i}$, for $i=(j-1)(q+1)+1, \ldots, j(q+1)$, gets at least one bit. This contradicts the fact that the total size of advice is at most $q$.

Therefore, the property does not hold. This means that there exists an integer $i \leq k$ such that for any non-empty subset $S_{i}$ of $\left\{1, \ldots, n^{\prime}\right\}$, there exists a $k$-tuple $\mathcal{S}$ containing $S_{i}$ as the $i$-th element such that, in the graph $G_{\mathcal{S}}$, the source has advice $s_{j}$, where $j=\left\lfloor\frac{i-1}{q+1}\right\rfloor$, and the subgraph $H_{i}$ receives no bit of advice. In other words, there exists an index $i$ and a subfamily $\mathcal{G}^{\prime}$ of $\mathcal{G}$ such that for each graph in $\mathcal{G}^{\prime}$ the source always receives the same string while the 
subgraph $H_{i}$ never receives any advice from the oracle; moreover, for any nonempty subset $S_{i}$ of $\left\{1, \ldots, n^{\prime}\right\}$, there exists a graph in $\mathcal{G}^{\prime}$ where the graph $H_{i}$ is constructed from $S_{i}$. Therefore, for this subgraph $H_{i}$, the situation is identical as if it were alone (the graph is directed) and as if there were no oracle. Since there are no directed cycles in the graph, no node can receive any feedback, and hence any broadcasting algorithm in such a graph is oblivious. Therefore, using the argument from the proof of Theorem 2.2. in [23], for some graph $H_{i}$ the time of informing node $k \cdot n^{\prime}+i$ is at least $n^{\prime}$. This implies that there exists a graph in $\mathcal{G}^{\prime}$ in which the algorithm does not achieve broadcast in time less than $n^{\prime}$. Since $n^{\prime} \geq n^{\delta}$ and $\frac{1}{2} \log k \geq c \log n$, for $c=\epsilon / 2$, this proves the theorem.

\subsection{Geometric Radio Networks}

We finally show that the large advice requirements established in the previous section do not hold in the more restricted class of geometric radio networks. Indeed, for these networks we have the following result which should be contrasted with Theorems 1 and 2 .

Theorem 3. For any positive constant $c$ let $\mathcal{G}$ be the class of geometric radio networks whose optimal broadcasting time is at most $c$. There exists an oracle which gives advice of constant size to the nodes of networks of class $\mathcal{G}$ and an algorithm using this advice, which performs broadcast in constant time $c^{\prime}$, for any network in class $\mathcal{G}$.

To prove Theorem 3 we will use the following construction. Fix the ranges $r_{1}<\ldots<r_{\rho}$. (Recall that both the number $\rho$ of ranges and the ranges themselves are constants.) Partition the plane into a mesh of squares of side $z=r_{1} / \sqrt{2}$, called tiles, with the bottom-left corner of one of them in $(0,0)$. Include the left and bottom sides and exclude the top and right sides from every square. Knowing its position, every node knows to which tile it belongs. The tile to which the source belongs is called central. Observe that any two nodes belonging to the same tile are within each other's range. For any positive integer $x$, the $x$-block is a square consisting of $B(x)=(2 x+1)^{2}$ tiles with the central tile in the center of this square.

A configuration of points in the plane yielding a geometric radio network with optimal broadcasting time at most $c$ must have the property that the most distant points are at distance at most $2 c r_{\rho}$ and hence all points are contained in a $d$-block, for some positive constant $d$. Take the smallest such integer $d$. Order all the $B(d)$ tiles of the $d$-block in a fixed way, giving them indices $1, \ldots, B(d)$ and then order the $p(d)=B(d)(B(d)-1)$ ordered pairs of these indices in a fixed way, giving them indices $1, \ldots, p(d)$. Let $\lambda(a, b)$ denote the index of the pair $(a, b)$, where $a, b$ are (indices of) distinct tiles.

Advice. We now describe the oracle, called Geometric Oracle in the sequel. Consider an ordered pair $(a, b)$ of distinct tiles of the $d$-block. If there is a pair $(u, v)$ of nodes in tiles $a$ and $b$, respectively, such that $v$ is in the range of $u$, 
choose one such a pair. The oracle gives advice $(\lambda(a, b), o u t)$ to $u$ and advice $(\lambda(a, b), i n)$ to $v$. Clearly, the same node can get many pieces of advice, however, for constant $d$, the total number of bits of advice is constant. Moreover, any node that received the above advice, gets additionally the integer $d$.

We now describe the algorithm using the advice obtained from Geometric Oracle. It uses global round numbers which are transmitted from node to node appended to the source message.

Algorithm GRN-Broadcasting-with-Advice. The algorithm lasts $1+2 p(d) B(d)$ rounds. After round 1 it is divided into $B(d)$ identical stages, each lasting $p(d)$ 2-round periods. The pseudo-code follows:

in round 1 the source transmits;

starting in round 2 , repeat $B(d)$ times procedure Stage

where Stage is the following subroutine:

if $u$ has advice $(i$, out $)$, for $1 \leq i \leq p(d)$, and got the source message then it transmits in the first round of period $i$ of this stage

if $v$ has advice $(i, i n)$, for $1 \leq i \leq p(d)$, and got the source message

then it transmits in the second round of period $i$ of this stage

Theorem 3 follows from the following lemma whose proof is omitted.

Lemma 3. Algorithm GRN-Broadcasting-with-Advice, using the Geometric Oracle, is correct and has constant running time.

\section{The General Algorithm}

In this section we design and analyze a broadcasting algorithm working for arbitrary radio networks, whose running time is inverse-proportional to the size of advice given to nodes. We prove the following theorem.

Theorem 4. For any $q \in O(n)$ there exists an oracle which gives advice of size $q$ to the nodes of a network and an algorithm using this advice, which performs broadcast in time $O\left(\frac{n D}{q} \log ^{3} n\right)$ in any n-node network with source eccentricity $D$.

We prove Theorem 4 by constructing an appropriate oracle and algorithm. First assume that $q \in O\left(D \log n+\log ^{2} n\right)$. In this case we can use the broadcasting algorithm from [11] running in time $O\left(n \log ^{2} D\right)$ without using any advice, since $O\left(n \log ^{2} D\right) \subseteq O\left(\frac{n D}{q} \log ^{3} n\right)$, for this range of $q$. Therefore, in the sequel, we can assume $q \geq 6\left(D \log n+\log ^{2} n\right)$.

Given the directed graph $G=(V, E)$ with source $s$, let $L_{1}, \ldots, L_{D}$ be BFS layers in $G$, i.e., sets of nodes at distance exactly $i$ from the source, for $1 \leq i \leq D$. Let $T$ be the smallest power of 2 greater or equal to $1152 \frac{n}{q} \log ^{2} n$. For each $1 \leq i \leq D-1$ we will need sets $L_{i}(j) \subseteq L_{i}$, for $j=\log T, \log T+1, \ldots,\left\lfloor\log \left|L_{i}\right|\right\rfloor$, such that for every such $j$ the following properties hold: 
(i) every node in $L_{i+1}$ having at least $2^{j}$ and less than $2^{j+1}$ neighbors in $L_{i}$, has at least 1 and at most $144 \log n$ neighbors in $L_{i}(j)$;

(ii) $\left|L_{i}(j)\right|<144\left|L_{i}\right| \log n / 2^{j}$.

The following lemma justifies the existence of such sets (the proof is omitted).

Lemma 4. There exist sets $L_{i}(j) \subseteq L_{i}$, for $j=\log T, \log T+1, \ldots,\left\lfloor\log \left|L_{i}\right|\right\rfloor$ with the above properties.

Advice. We now describe the advice given by the oracle. The advice given to the source consists of integers $N, n, q$ and of the sizes of layers $L_{1}, \ldots, L_{D}$. This can be encoded using $3 D \log n \leq q / 2$ bits of advice. Moreover, to every node in set $L_{i}(j)$, for $1 \leq i \leq D-1$ and $\log T \leq j \leq \log \left|L_{i}\right|$, the oracle gives the integer $j$. (Note that, since sets $L_{i}(j)$ are not necessarily disjoint, a node may get several integers as advice.) This costs a total of at most

$$
2 \cdot \sum_{i=1}^{D-1} \sum_{j=\log T}^{\left\lfloor\log \left|L_{i}\right|\right\rfloor}\left(144\left|L_{i}\right| \log n / 2^{j} \cdot \log j\right) \leq 4 \cdot 144 \cdot(n / T) \log ^{2} n \leq q / 2
$$

bits, by property (ii) of sets $L_{i}(j)$. Hence the total size of advice is at most $q$.

Algorithm Radio-Broadcasting-with-Advice. We now describe the algorithm using the above advice. It uses global round numbers which are transmitted from node to node appended to the source message. First we define the additional information attached to the source message. We will use the notion of a $(N, x)$ selective family. This is a family $\mathcal{F}$ of subsets of $\{1, \ldots, N\}$, such that, for any set $X \subseteq\{1, \ldots, N\}$ of size at most $x$, there exists a set $F \in \mathcal{F}$, for which $|F \cap X|=1$. For any $x$, fix a $(N, x)$-selective family $\mathcal{S}(N, x)$ of size $s(N, x)$. By [10] there exist $(N, x)$-selective families of size $O(x \log (N / x)) \subseteq O(x \log n)$, thus we can assume that $s(N, x) \leq b \cdot x \log n$ for some constant $b>0$. Fix an order $\left(F_{1}, \ldots, F_{s(N, x)}\right)$ of the family $\mathcal{S}(N, x)$. Knowing $T$, sizes $\left|L_{i}\right|$ of layers and the constant $b$, the source computes the sequence of rounds $t_{1}<\ldots<t_{D-1}$ recursively as follows:

$t_{0}=0, \quad t_{i+1}=t_{i}+s(N, T)+\left(\log \left|L_{i}\right|-\log T+1\right) \cdot s(N,\lceil 144 \log n\rceil)$, for $1 \leq i \leq D-1$.

Then the source broadcasts the source message together with the sequence $t_{1}, \ldots, t_{D-1}$ and $\left|L_{1}\right|, \ldots,\left|L_{D-1}\right|$ in round 0 . A node that receives this message for the first time in round $t$, where $t_{i-1}<t \leq t_{i}$ for some $1 \leq i \leq D-1$, waits till round $t_{i}$ and starts transmitting according to the $(N, T)$-selective family $\mathcal{S}(N, T)$, starting in round $t_{i}+1$ until round $t_{i}+s(N, T)$. More precisely, a node with label $u$ transmits in round $t_{i}+y$, if $u$ is in $F_{y}$, where $F_{y}$ is the $y$-th set of the family $\mathcal{S}(N, T)$. Additionally, if a node has the integer $j$ in its advice string then it transmits according to the family $\mathcal{S}(N,\lceil 144 \log n\rceil)$ in the time interval from $t_{i}+s(N, T)+(j-\log T) \cdot s(N,\lceil 144 \log n\rceil)+1$ to $t_{i}+s(N, T)+(j+1-$ $\log T) \cdot s(N,\lceil 144 \log n\rceil)$, for any $\log T \leq j \leq \log \left|L_{i+1}\right|$. A node without the integer $j$ in its advice string waits during this period. A node that receives the source message for the first time in round at most $t_{i}$ does not transmit in rounds beyond $t_{i+1}$. We omit the proof of the following lemma. 
Lemma 5. Assume $q \in O(n)$ and $q \geq 6\left(D \log n+\log ^{2} n\right)$. Our algorithm Radio-Broadcasting-with-Advice performs broadcasting in any n-node network with source eccentricity $D$ in time $O\left(\frac{n D}{q} \log ^{3} n\right)$ using at most $q$ bits of advice.

Since, as we noticed before, for $q \in O\left(D \log n+\log ^{2} n\right)$, the time $O\left(\frac{n D}{q} \log ^{3} n\right)$ of broadcasting can be achieved even without advice, Lemma 5 concludes the proof of Theorem 4 .

Corollary 1. For n-node networks with source eccentricity $D$ polylogarithmic in $n$, there exists advice of size o(n) sufficient to achieve polylog $(n)$ broadcasting time.

The above corollary should be contrasted with the lower bound from [10], were it is shown that (without advice) some $n$-node networks with source eccentricity $D$ require time $\Omega(n \log D)$.

\section{Conclusion}

We studied the impact of the size of information (advice) given to nodes of a radio network on the time of broadcasting. Our approach was quantitative, i.e., we were concerned with the total number of bits, as opposed to particular items of information, such as the knowledge of neighborhood, or of the size of the network, whose impact on broadcasting time was previously studied in the literature. While our algorithm is a first step towards grasping the trade-off between the size of advice and the time of broadcasting, establishing the exact trade-offs, for any number of bits of advice, remains an open problem. Its general formulation is: What is the minimum time to broadcast in radio networks, with advice of size $q$ ? A more specific question is: What is the minimum size of advice permitting to achieve broadcasting time $O(O p t(G))$ for any radio network $G$. We answered this question for networks with constant optimal time.

Establishing trade-offs between the size of advice and broadcasting time is also open for geometric radio networks. For these networks time $O(D)$, where $D$ is the eccentricity of the source, is optimal under full knowledge of the network. It is easy to show that $O\left(\min \left(n, D^{2}\right)\right)$ bits of advice are sufficient to achieve this time. Is this size of advice also necessary?

Another interesting problem is to compare the size of arbitrary advice permitting given broadcasting time with the size of advice of given type, e.g., concerning the immediate neighborhood. It was proved in [24] that giving to all nodes information about their immediate neighborhood (a total of $\Theta(|E| \log n$ ) bits) permits broadcasting in time $O\left(n^{2 / 3} \log n\right)$ in networks with source eccentricity 2. In [3] it was proved that time $\Omega(\sqrt{n})$ is necessary for these networks with this information. This should be contrasted with the algorithm from the present paper which, e.g., permits broadcasting in these networks in the same time $O\left(n^{2 / 3} \log n\right)$ using only $O\left(n^{1 / 3}\right.$ polylog $\left.(n)\right)$ bits of advice, provided that the advice is of non-restricted type. On the other hand, $O(\sqrt{n}$ polylog $(n))$ bits of advice suffice to beat time $\Theta(\sqrt{n})$ for these networks. These examples suggest 
that using advice of non-restricted type may be much more efficient than that of a particular type.

The paradigm of radio broadcasting with advice also suggests related problems for randomized algorithms: What is the minimum number of random bits provided to the nodes of a radio network of unknown topology that is sufficient to achieve randomized broadcasting in optimal expected time? The lower bound on the expected broadcasting time obtained by Kushilevitz and Mansour [28] can be directly applied to the class of graphs $G$ defined as follows: $G$ consists of three layers, the only directed connections are from a layer to the subsequent layer, the first layer consists of the source, and each node in the middle layer has at most one out-neighbor in the last layer. In view of the result from [28], the number of random bits provided to the system must be $\Omega(n \log n)$ in order to guarantee $O(\log n)$ expected time. By contrast, $O(n)$ bits of advice suffice to achieve constant deterministic broadcast time for these networks. This means that randomization is sometimes more costly than advice by a logarithmic factor, in terms of the number of bits. The precise trade-off between randomized broadcasting time and the number of random bits used by a distributed randomized broadcasting algorithm remains open.

\section{References}

1. N. Alon, A. Bar-Noy, N. Linial, D. Peleg, A lower bound for radio broadcast, Journal of Computer and System Sciences 43 (1991), 290-298.

2. R. Bar-Yehuda, O. Goldreich, A. Itai, On the time complexity of broadcast in radio networks: an exponential gap between determinism and randomization. Journal of Computer and System Sciences 45 (1992), 104-126.

3. C. Brito, E. Gafni, S. Vaya, An information theoretic lower bound for broadcasting in radio networks, Proc. 21st Annual Symposium on Theoretical Aspects of Computer Science, (STACS 2004), LNCS 2996, 534-546.

4. D. Bruschi, M. Del Pinto, Lower bounds for the broadcast problem in mobile radio networks. Distributed Computing 10 (1997), 129-135.

5. I. Chlamtac, S. Kutten, On broadcasting in radio networks - problem analysis and protocol design. IEEE Transactions on Communications 33 (1985), 1240-1246.

6. I. Chlamtac, O. Weinstein, The wave expansion approach to broadcasting in multihop radio networks, IEEE Transactions on Communications 39 (1991), 426-433.

7. B. Chlebus, L. Gąsieniec, A. Gibbons, A. Pelc, W. Rytter, Deterministic broadcasting in unknown radio networks. Distributed Computing 15 (2002), 27-38.

8. B. Chlebus, L. Gąsieniec, A. Östlin, J.M. Robson, Deterministic radio broadcasting. Proc. 27th International Colloquium on Automata, Languages and Programming (ICALP 2000), LNCS 1853, 717-728.

9. M. Chrobak, L. Ga̧sieniec, D. Kowalski, The wake-up problem in multi-hop radio networks, Proc. 15th ACM-SIAM Symposium on Discrete Algorithms (SODA 2004), $985-993$.

10. A.E.F. Clementi, A. Monti, R. Silvestri, Selective families, superimposed codes, and broadcasting on unknown radio networks, Proc. 12th Ann. ACM-SIAM Symposium on Discrete Algorithms (SODA 2001), 709-718. 
11. A. Czumaj, W. Rytter, Broadcasting algorithms in radio networks with unknown topology. Proc. 44th Symposium on Foundations of Computer Science (FOCS 2003) 492-501.

12. G. De Marco, Distributed broadcast in unknown radio networks. In Proc. 19th ACM-SIAM Symp. on Discrete Algorithms (SODA 2008).

13. A. Dessmark, A. Pelc, Broadcasting in geometric radio networks, Journal of Discrete Algorithms 5 (2007), 187-201.

14. K. Diks, E. Kranakis, D. Krizanc, A. Pelc, The impact of knowledge on broadcasting time in linear radio networks, Theoretical Computer Science 287 (2002), 449-471.

15. M. Elkin, G. Kortsarz, Improved broadcast schedule for radio networks. Proc. 16th ACM-SIAM Symposium on Discrete Algorithms (SODA 2005).

16. Y. Emek, L. Gasieniec, E. Kantor, A. Pelc, D. Peleg, C. Su, Broadcasting time in UDG radio networks with unknown topology, Proc. 26th Ann. ACM Symposium on Principles of Distributed Computing (PODC 2007), 195-204.

17. P. Fraigniaud, D. Ilcinkas, A. Pelc, Oracle size: a new measure of difficulty for communication problems, Proc. 25th Ann. ACM Symposium on Principles of Distributed Computing (PODC 2006), 179-187.

18. P. Fraigniaud, D. Ilcinkas, A. Pelc, Tree exploration with an oracle, Proc. 31st International Symposium on Mathematical Foundations of Computer Science (MFCS 2006), LNCS 4162, 24-37.

19. P. Fraigniaud, D. Ilcinkas, C. Gavoille, A. Pelc, Distributed computing with advice: Information sensitivity of graph coloring, Proc. 34th International Colloquium on Automata, Languages and Programming (ICALP 2007), LNCS 4596, 231-242.

20. P. Fraigniaud, A. Korman, E. Lebhar, Local MST computation with short advice, Proc. 19th Annual ACM Symposium on Parallelism in Algorithms and Architectures (SPAA 2007), 154-160.

21. I. Gaber, Y. Mansour, Centralized broadcast in multihop radio networks. Journal of Algorithms 46 (2003), 1-20.

22. L. Gasieniec, D. Peleg, Q. Xin, Faster communication in known topology radio networks, Proc. 24th Annual ACM Symposium on Principles Of Distributed Computing (PODC 2005), 129-137.

23. L. Gassieniec, A. Pelc, D. Peleg, The wakeup problem in synchronous broadcast systems, SIAM Journal on Discrete Mathematics 14 (2001), 207-222.

24. D. Kowalski, A. Pelc, Time of deterministic broadcasting in radio networks with local knowledge, SIAM Journal on Computing 33 (2004), 870-891.

25. D. Kowalski, A. Pelc, Time complexity of radio broadcasting: adaptiveness vs. obliviousness and randomization vs. determinism, Theoretical Computer Science 333 (2005), 355-371.

26. D. Kowalski, A. Pelc, Broadcasting in undirected ad hoc radio networks, Distributed Computing 18 (2005), 43-57.

27. D. Kowalski, A. Pelc, Optimal deterministic broadcasting in known topology radio networks, Distributed Computing 19 (2007), 185-195.

28. E. Kushilevitz, Y. Mansour, An $\Omega(D \log (N / D))$ lower bound for broadcast in radio networks. SIAM Journal on Computing 27 (1998) 702-712.

29. A. Sen, M. L. Huson, A new model for scheduling packet radio networks, Proc. 15th Annual Joint Conference of the IEEE Computer and Communication Societies (IEEE INFOCOM 1996), 1116 - 1124.

30. D. Soguet, N. Nisse, Graph searching with advice, Proc. 14th International Colloquium on Structural Information and Communication Complexity (SIROCCO 2007), LNCS 4474, 51-65. 